TRANSACTIONS OF THE

AMERICAN MATHEMATICAL SOCIETY

Volume 364, Number 3, March 2012, Pages 1395-1411

S 0002-9947(2011)05410-3

Article electronically published on November 7, 2011

\title{
APPROACH TO ARTINIAN ALGEBRAS VIA NATURAL QUIVERS
}

\author{
FANG LI AND ZONGZHU LIN
}

\begin{abstract}
Given an Artinian algebra $A$ over a field $k$, there are several combinatorial objects associated to $A$. They are the diagram $D_{A}$ as defined by Drozd and Kirichenko, the natural quiver $\Delta_{A}$ defined by Li (cf. Section 2), and a generalized version of $k$-species $\left(A / r, r / r^{2}\right)$ with $r$ being the Jacobson radical of $A$. When $A$ is splitting over the field $k$, the diagram $D_{A}$ and the well-known Ext-quiver $\Gamma_{A}$ are the same. The main objective of this paper is to investigate the relations among these combinatorial objects and in turn to use these relations to give a characterization of the algebra $A$.
\end{abstract}

\section{INTRODUCTION}

1.1. Given an Artinian algebra $A$ over a field $k$, there are several combinatorial objects associated to $A$. They are the diagram $D_{A}$ as defined in [DK], the natural quiver $\Delta_{A}$ defined in Li (cf. Section 2), and a generalized version of $k$-species $\left(A / r, r / r^{2}\right)$ with $r$ being the Jacobson radical of $A$. When $A$ is splitting over the field $k$, the diagram $D_{A}$ and the well-known Ext-quiver $\Gamma_{A}$ are the same. The main objective of this paper is to investigate the relations among these combinatorial objects and in turn to use these relations to give a characterization of the algebra A.

For a given Artinian $k$-algebra $A$, let $\left\{S_{1}, S_{2}, \cdots, S_{s}\right\}$ be the complete set of nonisomorphic irreducible $A$-modules. Set $D_{i}=\operatorname{End}_{A}\left(S_{i}\right)$, which is a division ring, and $\operatorname{Ext}_{A}^{1}\left(S_{i}, S_{j}\right)$, which is a $D_{i}$ - $D_{j}$-bimodule. $A$ is said to split over the ground field $k$, or say, to be $k$-splitting, if $\operatorname{dim}_{k} \operatorname{End}_{A}\left(S_{i}\right)=1$ (i.e., $D_{i}=k$ for all irreducible $A$-modules $\left.S_{i}\right)$. Recall that a quiver is a finite directed graph $\Gamma=\left(\Gamma_{0}, \Gamma_{1}\right)$ with vertex set $\Gamma_{0}$ and arrow set $\Gamma_{1}$. For a $k$-splitting algebra $A$, one can define a finite quiver $\Gamma_{A}$ called the Ext-quiver of $A$ by setting $\Gamma_{0}=\{1,2, \cdots, s\}$ and with $m_{i j}=\operatorname{dim}_{k}\left(\operatorname{Ext}_{A}^{1}\left(S_{i}, S_{j}\right)\right)$ being the number of arrows from $i$ to $j$.

There is another way to characterize the Ext-quiver for a $k$-splitting algebra Artinian algebra. By [ARS and [Liu, when $A$ is a finite dimensional algebra over a field $k$ and $1=\varepsilon_{1}+\cdots+\varepsilon_{s}$ is a decomposition of 1 into a sum of primitive orthogonal idempotents, then we can re-index $\left\{S_{1}, S_{2}, \cdots, S_{s}\right\}$ such that $S_{i} \cong A \varepsilon_{i} / r \varepsilon_{i}$ where $r$ is the radical of $A$, and moreover, $\operatorname{dim}_{k} \operatorname{Ext}_{A}\left(S_{i}, S_{j}\right)=\operatorname{dim}_{k}\left(\varepsilon_{j} r / r^{2} \varepsilon_{i}\right)$.

Received by the editors August 14, 2009 and, in revised form, May 18, 2010.

2010 Mathematics Subject Classification. Primary 16G10, 16G20; Secondary 16P20, 13E10.

This project was supported by the National Natural Science Foundation of China (No. 10871170) and the Natural Science Foundation of Zhejiang Province of China (No. D7080064).

The second author was supported in part by an NSA grant and the NSF I/RD program.

(C)2011 American Mathematical Society Reverts to public domain 28 years from publication 
If $A$ is basic and $k$-splitting, then $A$ is a quotient of the path algebra of $\Gamma_{A}$. The properties of this quiver $\Gamma_{A}$, in particular, its relation to the representations of $A$, has been extensively studied in the field of representations of algebras. $\Gamma_{A}$ is invariant under Morita equivalence, i.e., if $A$ and $B$ are two Morita equivalent $k$-algebras, then $\Gamma_{A}$ is isomorphic to $\Gamma_{B}$.

If $A$ is not basic, it is no longer isomorphic to a quotient of the path algebra $k \Gamma_{A}$. It is discussed in this paper and in [Li] as to how to get the analogue of the Gabriel theorem in this case.

1.2. In recent years, geometric methods have been heavily used in representation theory of algebras. To each finite dimensional algebra $A$ over an algebraically closed field $k$, one can associate a sequence of algebraic varieties $\operatorname{Mod}_{k}^{d}(A)(d=1,2,3 \ldots)$ as closed subvarieties of the affine spaces $k^{d \times d}$. The association of the varieties depends on the presentation of the algebra $A$ using finitely many generators and finitely many relations. In $[\mathrm{B}$, it is proved that two algebras $A$ and $B$ are isomorphic if and only if the associated varieties $\operatorname{Mod}_{k}^{d}(A)$ and $\operatorname{Mod}_{k}^{d}(B)$ are isomorphic as $G L_{d}(k)$-varieties. Thus having a more standard presentation of the algebra $A$ will help with studying these varieties. The purpose of this paper is to explore relations of the Ext-quiver of $A$ and the natural quiver which will be defined in Section 2 . The natural quiver will have fewer arrows than the Ext-quiver when the algebra $A$ is not basic. Natural quivers are not invariant under the Morita equivalence and are much closer to reflect the structure of the algebra rather than just its module category. There are numerous cases, even in representation theory, where one needs the structure of the algebras. For example, the character values of finite groups in a block cannot be preserved through Morita equivalence.

1.3. The paper is organized as follows. In Section 2 we recall the definition of the natural quiver $\Delta_{A}$ of an Artinian $k$-algebra $A$ and provide a precise relation with the Ext-quiver $\Gamma_{A}$ when the algebra $A$ is splitting over the ground field $k$. In Section 3 we prove in Theorem 3.4 that any Artinian algebra, which is splitting over its radical, is a quotient of the generalized path algebra of its natural quiver associated to $A / r$. This gives a presentation of the algebra $A$. Although there is always a surjective algebra homomorphism from the path algebra of the natural quiver $\Delta_{A}$ to the tensor algebra $T_{A / r}\left(r / r^{2}\right)$, the above surjective map to $A$ does not always factor through $T_{A / r}\left(r / r^{2}\right)$ (Example 3.5). There have been numerous generalizations of Wedderburn-Malcev theorems to characterize an Artinian algebra that is splitting over its radical. By using the generalized path algebra of the natural quiver, we give another characterization of an Artinian algebra $A$ which is splitting over its radical; see Corollary 3.6. Moreover, we discuss the relations among the natural quiver and the Ext-quiver of an Artinian algebra and the associated generalized path algebra (see the figure at the end of Section 4) and, furthermore, their relationship with the diagram of an Artinian algebra as defined in [DK. The main results are the formulae in Theorems 2.2 and 5.3. As an application, in Section 5, we discuss the relationship between the diagram and natural quiver of an Artinian algebra that is not splitting over the ground field. In Section 6 we prove that a (not necessarily basic) hereditary Artinian algebra which is splitting over its radical is isomorphic to the generalized path algebras of its natural quiver, provided the defining ideal as described in Theorem 3.4 does not intersect with the arrow space (see Theorem 6.5). 


\section{The Relation BetWEen NATURAL QUiver AND EXT-QUiver}

Suppose that $A$ is a left Artinian $k$-algebra and $r=r(A)$ is its Jacobson radical.

Write $A / r=\bigoplus_{i=1}^{s} A_{i}$, where $A_{i}$ are two-sided simple ideals of $A / r$. Such a decomposition of $A / r$ is also called a block decomposition of the algebra $A / r$. Then, $r / r^{2}$ is an $A / r$-bimodule. Let ${ }_{i} M_{j}=A_{i} \cdot r / r^{2} \cdot A_{j}$, which is finitely generated as an $A_{i}-A_{j}$-bimodule for each pair $(i, j)$.

For two rings $A$ and $B$, and a finitely generated $A$ - $B$-bimodule $M$, define $\operatorname{rk}_{A, B}(M)$ to be the minimal number of generators of $M$ as an $A$-B-bimodule among all generating sets. As a convention, we always denote $\operatorname{rk}_{A, B}(0)=0$.

The isomorphism classes of irreducible $A$-modules is indexed by the set $\Delta_{0}=$ $\{1, \cdots, s\}$ corresponding to the set of blocks of $A / r$. We now define the natural quiver $\Delta_{A}=\left(\Delta_{0}, \Delta_{1}\right)$ with $\Delta_{0}$ being the vertex set and, for $i, j \in \Delta_{0}, t_{i j}=$ $\operatorname{rk}_{A_{j}, A_{i}}\left({ }_{j} M_{i}\right)$ being the number of arrows from $i$ to $j$ in $\Delta_{A}$. Obviously, there is no arrow from $i$ to $j$ if ${ }_{j} M_{i}=0$. This quiver $\Delta_{A}=\left(\Delta_{0}, \Delta_{1}\right)$ is called the natural quiver of $A$.

The notion of a natural quiver was first introduced in [Li], where the aim was to use the generalized path algebra from the natural quiver of an Artinian algebra $A$ to characterize $A$ through the generalized Gabriel theorem. The advantage of a generalized path algebra is that valued quiver information is already encoded in the generalized path algebras. In the language of Kontsevich and Soilbelman [KY], the Gabriel-type algebra cannot be stated as an affine non-commutative scheme which can be embedded into a thin scheme in a sense that they are "infinitesimally" isomorphic. The aim of this paper will be to find a "smallest" embedding.

For a quiver $Q=\left(Q_{0}, Q_{1}\right)$, a sub-quiver $Q^{\prime}$ of $Q$ is called dense if $\left(Q^{\prime}\right)_{0}=Q_{0}$, and for any vertices $i, j$, there exists an arrow from $i$ to $j$ in $Q^{\prime}$ if and only if there exists an arrow from $i$ to $j$ in $Q$.

When $A$ is splitting over the ground field $k$, then $A_{i} \cong M_{n_{i}}(k)$ and the irreducible module $S_{i}$ has $k$-dimension $n_{i}$. In this case the Ext-quiver $\Gamma_{A}$ of $A$ is defined (cf. Section 1.1). It is proved in [Liu, Prop. 7.4.3] that

$$
t_{i j} \leq m_{i j} \leq n_{i} n_{j} t_{i j}
$$

In addition, if $A$ is basic, then $\Delta_{A}=\Gamma_{A}$ as discussed in Section 1.1. In general these two constructions will give two different quivers if $A$ is not basic. The following results were proved in [LC].

Proposition 2.1. Let $A$ be a $k$-splitting Artinian $k$-algebra over a field $k$ and let $B$ be the corresponding basic algebra of $A$. Then:

(i) $\Gamma_{A}=\Gamma_{B}$;

(ii) $\Gamma_{B}=\Delta_{B}$;

(iii) $\Delta_{A}$ is a dense sub-quiver of $\Gamma_{A}$, and thus a dense sub-quiver of $\Gamma_{B}$ and $\Delta_{B}$

Now, we will just give the exact relation between $t_{i j}$ and $m_{i j}$ when the algebra $A$ is splitting over $k$. First, we recall that for any real number $a$, the ceiling of $a$ is defined to be

$$
\lceil a\rceil=\min \{n \in \mathbb{Z} \mid n \geq a\} .
$$

Theorem 2.2. Let $A$ be an Artinian $k$-algebra $A$ which is splitting over $k$. Assume $\Gamma_{A}$ is the Ext-quiver of $A$ and $\Delta_{A}$ is the natural quiver of $A$. Then $t_{i j}=\left\lceil\frac{m_{i j}}{n_{i} n_{j}}\right\rceil$ for $i, j \in\left(\Gamma_{A}\right)_{0}=\left(\Delta_{A}\right)_{0}$. Here $m_{i j}$ is the number of arrows from $i$ to $j$ in $\Gamma_{A}$. 
Proof. The proof involves computing a minimal generating set of ${ }_{i} M_{j}=A_{i} \cdot\left(r / r^{2}\right)$. $A_{j}$ as $A_{i}-A_{j}$-bimodules. We first note that both $A_{i}$ and $A_{j}$ are simple $k$-algebras and are split over $k$. Hence $A_{i} \otimes_{k} A_{j}^{o p}$ is a also a simple central $k$-algebra, isomorphic to $M_{n_{i} n_{j}}(k)$. Since $r / r^{2}$ is a semisimple left $A_{i}$-module and a semisimple right $A_{j^{-}}$ module, ${ }_{i} M_{j}$ is a semisimple $A_{i} \otimes_{k} A_{j}^{o p}$-module with simple components isomorphic to $S_{i} \otimes_{k} S_{j}^{o p}$. Here $S_{j}^{o p}$ is the right irreducible $A_{j}$-module. Let $e_{k l}^{i}$ be the standard matrix basis elements of $A_{i}=M_{n_{i}}(k)$. Then $\operatorname{dim}_{k} \operatorname{Ext}_{A}^{1}\left(S_{i}, S_{j}\right)=\operatorname{dim}_{k} e_{11}^{i} r / r^{2} e_{11}^{j}$. We claim that ${ }_{i} M_{j}$ has exactly $\operatorname{dim}_{k} e_{11}^{i} r / r^{2} e_{11}^{j}$ many $A_{i} \otimes_{k} A_{j}^{o p}$-composition factors. Indeed $e_{11}^{i} \otimes e_{11}^{j}$ is a primitive idempotent of $A_{i} \otimes_{k} A_{j}^{o p}$. Now the theorem follows from the following lemma for simple Artinian rings.

Lemma 2.3. Let $R=M_{n}(D)$ be the ring of all $n \times n$-matrices with entries in a division ring $D$ and let $M$ be an $R$-module. Let $L=D^{n}$ be the natural irreducible $R$-module of column vectors. Then:

(i) $M$ is a semisimple $R$-module isomorphic to $L^{\oplus m}=L \oplus L \oplus \cdots \oplus L$, where $m=\operatorname{dim}_{D}\left(e_{11} M\right)$;

(ii) $M$ can be generated by $\left\lceil\frac{m}{n}\right\rceil$ many elements over $R$ but cannot be generated by a fewer number of elements.

Proof. Since $R$ is simple, all finitely generated $R$-modules are semisimple. Note that every irreducible $R$-module is isomorphic to $L=D^{n}$. Then (i) follows from the fact that $\operatorname{dim}_{D} e_{11} L=1$. To show (ii), we note that $R=\bigoplus_{s=1}^{n} R e_{s s} \cong L^{\oplus n}$ as a left $R$ module. If $M$ is generated by $t$-many elements, then $M$ is a quotient of $R^{\oplus t}$ as a left $R$-module. Hence, $M$ has at most $t n$ composition factors counting multiplicity, i.e., $m \leq t n$ and $\left\lceil\frac{m}{n}\right\rceil \leq t$. Thus $M$ cannot be generated by less than $\left\lceil\frac{m}{n}\right\rceil$ many elements. On the other hand, let $N$ be any $R$-module of length $p \leq n$. Then $N$ is isomorphic to $L^{\oplus p}$. There is a surjective homomorphism $\phi: R \rightarrow N$ of left $R$-modules. By writing $m=t n+s$, with $0 \leq s<n$, we have $\left\lceil\frac{m}{n}\right\rceil=t$ if $s=0$ and $\left\lceil\frac{m}{n}\right\rceil=t+1$ if $s>0$. Hence we can construct a surjective homomorphism $R^{\left\lceil\frac{m}{n}\right\rceil} \rightarrow L^{\oplus t n+s}=\left(L^{\oplus n}\right)^{\oplus t} \oplus L^{\oplus s}$. Hence $M$ is generated by $\left\lceil\frac{m}{n}\right\rceil$ many elements.

As we note in Proposition 2.1 (ii), the Ext-quiver and the natural quiver of a finite dimensional basic algebra coincide with each other. As an application of Theorem 2.2, we give an example which means that the coincidence is also possible to happen for some non-basic algebras.

Example 2.4. Let $k$ be a field of characteristic different from 2 and let $Q$ be the quiver:

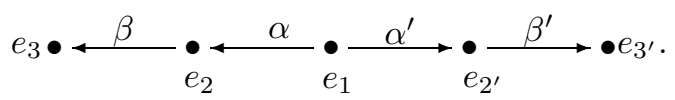

Let $\Lambda=k Q$ be the path algebra of $Q$ and $G=\langle\sigma\rangle$ be the automorphism group of $Q$ of order 2. Then $\sigma$ defines a $k$-algebra automorphism of $k Q$. Now, we consider the Ext-quiver and the natural quiver of the skew group algebra $\Lambda G$ (see [ARS]).

Let $r$ be the Jacobson radical of $\Lambda$. By Proposition 4.11 in ARS, $(r \Lambda G)$ is the Jacobson radical of $\Lambda G$. It is easy to see that $(\Lambda G) /(r \Lambda G) \cong(\Lambda / r) G$. On Page 84 of ARS, it was given that $(\Lambda / r) G \cong A_{1} \times A_{2} \times A_{3} \times A_{4}=k \times k \times\left(\begin{array}{cc}k & k \\ k & k\end{array}\right) \times\left(\begin{array}{cc}k & k \\ k & k\end{array}\right)$ as algebras and that the associated basic algebra $B$ is obtained in the reduced form 
from $\Lambda G$, which is Mortia equivalent to $\Lambda G$. Moreover, it was proved in [ARS] that $B$ is isomorphic to the path algebra of the following quiver

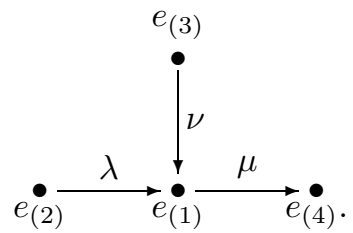

This quiver is just the Ext-quiver $\Gamma_{\Lambda G}$ of $\Lambda G$. Therefore, all $m_{i j}=0$ or 1 . For $i=1,2,3,4, \operatorname{dim}_{k} A_{i}=n_{i}^{2}$, where $n_{1}=n_{2}=1, n_{3}=n_{4}=2$. By Theorem 2.2. $t_{i j}=\left\lceil\frac{m_{i j}}{n_{i} n_{j}}\right\rceil$. Then, for each pair $(i, j)$, we have $t_{i j}=m_{i j}=0$ or 1 . Therefore, the natural quiver $\Delta_{\Lambda G}$ is equal to the Ext-quiver $\Gamma_{\Lambda G}$.

\section{Algebras SPlitting over Radicals}

The concept of a generalized path algebra was introduced early in CL]. Here we review a different but equivalent definition.

Given a quiver $Q=\left(Q_{0}, Q_{1}\right)$ and a collection of $k$-algebras $\mathcal{A}=\left\{A_{i} \mid i \in Q_{0}\right\}$, let $e_{i} \in A_{i}$ be the identity. Let $A_{0}=\prod_{i \in Q_{0}} A_{i}$ be the direct product $k$-algebra. Note that $e_{i}$ are orthogonal central idempotents of $A_{0}$.

For $i, j \in Q_{0}$, let $\Omega(i, j)$ be the subset of arrows in $Q_{1}$ from $i$ to $j$. Define $A_{i} \Omega(i, j) A_{j}$ to be the free $A_{i}$ - $A_{j}$-bimodule (in the category of $k$-vector spaces) with basis $\Omega(i, j)$. This is the free $A_{i} \otimes_{k} A_{j}^{o p}$-module over the set $\Omega(i, j)$.

Then $M=\bigoplus_{i, j} A_{i} \Omega(i, j) A_{j}$ is an $A_{0}$ - $A_{0}$-bimodule. The generalized path algebra is defined to be the tensor algebra

$$
T_{A_{0}}(M)=\bigoplus_{n=0}^{\infty} M^{\otimes A_{0} n} .
$$

Here $M^{\otimes_{A_{0}} n}=M \otimes_{A_{0}} M \otimes_{A_{0}} \cdots \otimes_{A_{0}} M$ and $M^{\otimes_{A_{0}} 0}=A_{0}$. We denote the generalized path algebra by $k(Q, \mathcal{A})$. Elements in $M^{\otimes A_{0} n}$ are called virtual $\mathcal{A}$-paths of length $n$. In cases of path algebras, virtual $\mathcal{A}$-paths are linear combinations of $\mathcal{A}$-paths of equal length. We denote $J=\bigoplus_{n=1}^{+\infty} M^{\otimes A_{0} n}$. Then $k(Q, \mathcal{A}) / J \cong A_{0}$.

The generalized path algebra has the following universal mapping property. For any $k$-algebra $B$ with any $k$-algebra homomorphism $\phi_{0}: A_{0} \rightarrow B$ (thus making $B$ an $A_{0}$ - $A_{0}$-bimodule) and any $A_{0}$ - $A_{0}$-bimodule homomorphism $\phi_{1}: M \rightarrow B$, there is a unique $k$-algebra homomorphism $\phi: k(Q, \mathcal{A}) \rightarrow B$ extending $\phi_{0}$ and $\phi_{1}$.

This definition is equivalent to the original definition in $[\mathrm{CL}$ and has the advantage of the above-mentioned universal mapping property. As a matter of fact, the classical path algebras are the special cases by taking $A_{i}=k$. We are more interested in the case when $A_{i}$ are simple $k$-algebras, in particular when all $A_{i}$ are central simple algebras. The generalized path algebra $k(Q, \mathcal{A})$ is called normal if all $A_{i}$ are simple $k$-algebras.

A $k$-algebra $A$ is said to be splitting over its radical $r$ if there is a $k$-algebra homomorphism $\rho: A / r \rightarrow A$ such that $\pi \circ \rho=\operatorname{Id}_{A / r}$. For example, if $A / r$ is separable and $A$ is Artinian, then $A$ is always splitting over $r$ as a result of the Wedderburn-Malcev theorem (and many generalizations in the literature $[\mathrm{P}]$ ). Note that a normal generalized path algebra $k(Q, \mathcal{A})$ with an acyclic (no oriented cycles) quiver $Q$ is always splitting over its $\operatorname{radical} \operatorname{rad}(k(Q, \mathcal{A}))=J$. This equality fails in general for normal generalized path algebras. 
Proposition 3.1. Let $Q$ be a finite quiver and $k(Q, \mathcal{A})$ be a normal generalized path algebra with each $A_{i}$ being finite dimensional. If $I$ is an ideal of $k(Q, \mathcal{A})$ with $J^{s} \subseteq I \subset J$ for some positive integer $s$, then $k(Q, \mathcal{A}) / I$ is a finite-dimensional algebra and $\operatorname{rad}(k(Q, \mathcal{A}) / I)=J / I$.

Proof. Since $Q$ is a finite quiver and $A_{i}$ are finite dimensional, we have that $M^{\otimes_{A_{0}} n}$ in (11) is finite dimensional over $k$ for all $n$. Hence $k(Q, \mathcal{A}) / J^{s}$ is also finite dimensional. It follows that $k(Q, \mathcal{A}) / I$ is finite dimensional. On the other hand, $J / I$ is a nilpotent ideal of $k(Q, \mathcal{A}) / I$ with $(J / I)^{s}=0$ and $(k(Q, \mathcal{A}) / I) /(J / I) \cong k(Q, \mathcal{A}) / J \cong$ $\bigoplus_{i \in Q_{0}} A_{i}$ is a semisimple algebra. This implies $\operatorname{rad}(k(Q, \mathcal{A}) / I)=J / I$.

We remark that the finite dimensionality of $A_{i}$ over $k$ cannot be removed. For example, take the Dynkin quiver $Q$ of type $A_{2}$ with two vertices $\{1,2\}$ and one arrow $\alpha$ from 1 to 2 . Let $A_{1}$ and $A_{2}$ be two infinite-dimensional field extensions of $k$. Although $A_{1}$ and $A_{2}$ are two simple $k$-algebras, the path algebra $k(Q, \mathcal{A})$ is neither left nor right Artinian since

$$
A_{2} \alpha V=\left\{\sum_{i} a_{i} \alpha b_{i}: a_{i} \in A_{2}, b_{i} \in V\right\}
$$

is a left ideal of $k(Q, \mathcal{A})$ for any vector subspace $V$ of $A_{1}$.

In this section we will show that every Artinian algebra $A$ which is splitting over its radical will be a quotient of a generalized path algebra of its natural quiver.

For an Artinian algebra $A$ with radical $r$, let $\Delta_{A}$ be its natural quiver and $A / r=\bigoplus_{i \in\left(\Delta_{A}\right)_{0}} A_{i}$, where all $A_{i}$ are simple algebras. Denote $\mathcal{A}=\left\{A_{i}: i \in \Delta_{0}\right\}$. Then, the generalized path algebra $k\left(\Delta_{A}, \mathcal{A}\right)$ is called the associated generalized path algebra of $A$.

In $[\mathrm{LC}$, we introduce the following notion of a Gabriel-type algebra.

Definition 3.2. Let $A$ be an Artinian $k$-algebra and $k\left(\Delta_{A}, \mathcal{A}\right)$ be its associated generalized path algebra. $A$ is said to be of Gabriel type for a generalized path algebra if $A \cong k\left(\Delta_{A}, \mathcal{A}\right) / I$ for some ideal $I$ of $k\left(\Delta_{A}, \mathcal{A}\right)$ contained in $J$. We call $I$ the defining ideal of $A$.

In $[\mathrm{Li}$ and $[\mathrm{LC}$, the conditions for certain Artinian algebras to be of Gabriel type were discussed under different assumptions. Here we give a more general description.

Theorem 3.3. Let $A$ be a Gabriel-type Artinian algebra for a generalized path algebra over a field $k$ such that $A \cong k\left(\Delta_{A}, \mathcal{A}\right) / I$ with the ideal $I$ of $k\left(\Delta_{A}, \mathcal{A}\right)$ satisfying $I \subset J$. Then, $A$ is splitting over its radical $r$.

Proof. The assumption $I \subseteq J$ implies that

$$
\left(k\left(\Delta_{A}, \mathcal{A}\right) / I\right) /(J / I) \cong k\left(\Delta_{A}, \mathcal{A}\right) / J \cong A_{1} \oplus \cdots \oplus A_{p} \cong A / r
$$

is semisimple; then $\operatorname{rad} A \cong \operatorname{rad}\left(k\left(\Delta_{A}, \mathcal{A}\right) / I\right)=J / I$. We have $A_{1} \oplus \cdots \oplus A_{p} \subset$ $k\left(\Delta_{A}, \mathcal{A}\right)$ and $\left(A_{1} \oplus \cdots \oplus A_{p}\right) \cap I \subset\left(A_{1} \oplus \cdots \oplus A_{p}\right) \cap J=0$. Hence, we get $A_{1} \oplus \cdots \oplus A_{p} \stackrel{\varepsilon}{\hookrightarrow} k\left(\Delta_{A}, \mathcal{A}\right) / I \stackrel{\eta}{\rightarrow}\left(k\left(\Delta_{A}, \mathcal{A}\right) / I\right) /(J / I) \cong k\left(\Delta_{A}, \mathcal{A}\right) / J \cong A_{1} \oplus \cdots \oplus A_{p}$, which implies $\eta \varepsilon=1$. Therefore, $A$ is splitting over $r$.

In fact, in Theorem 8.5.4 of DK, it was proven that, for a finite-dimensional algebra $A$ with radical $r$, if the quotient algebra $A / r$ is separable, then $A$ is isomorphic to a quotient algebra of the tensor algebra $T_{A / r}\left(r / r^{2}\right)$ by an ideal $I$ such 
that $J^{s} \subset I \subset J^{2}$ for some positive integer $s$. The separability condition plays two roles in the proof. First, it guarantees the Wedderburn-Malcev theorem to get

(a) $A$ is splitting over its radical.

Secondly, the seperability also implies that

(b) $r / r^{2}$ is isomorphic to a direct summand of $r$ as an $A_{0}$ - $A_{0}$-bimodule.

It turns out that the properties (a) and (b) together are equivalent to the existence of the surjective map $\phi$ in the theorem.

It is proved in Li] that there always exists a surjective homomorphism of algebras $\pi: k\left(\Delta_{A}, \mathcal{A}\right) \rightarrow T_{A / r}\left(r / r^{2}\right)$. This can be seen from the universal property of $k\left(\Delta_{A}, \mathcal{A}\right)$. Hence any Artinian algebra $A$ with separable quotient $A / r$ is isomorphic to a quotient algebra of $k\left(\Delta_{A}, \mathcal{A}\right)$ by an ideal $I$ as in Theorem 3.3. The difference between the points given in this paper and those in $\mathrm{Li}$ is that the algebra $A$ is splitting over its radical and the given ideal $I$ is included in $J$ but not in $J^{2}$. The following theorem is an improvement of the result in $\mathrm{Li}$.

Theorem 3.4. Let $A$ be an Artinian k-algebra such that $A$ is splitting over its radical. Then there is a surjective algebra homomorphism $\phi: k\left(\Delta_{A}, \mathcal{A}\right) \rightarrow A$ with $J^{s} \subseteq \operatorname{ker}(\phi) \subseteq J$ for some positive integer $s$.

Proof. Let $A / r=A_{0}=\bigoplus_{i \in \Delta_{0}} A_{i}$. Then $A_{0}$ is semisimple and $r / r^{2}$ is a semisimple $A_{0}$-module. Since $A$ splits over $r$, we can regard $A_{0}$ as a subalgebra of $A$, and hence $r$ is an $A_{0}-A_{0}$-bimodule under the multiplication in $A$ and the quotient map ${ }^{-}: r \rightarrow r / r^{2}$ is a homomorphism of $A_{0}$ - $A_{0}$-bimodules. We will use this property in the following argument.

For each pair $(i, j)$, let ${ }_{i} M_{j}=A_{i}\left(r / r^{2}\right) A_{j}$. Let $X_{i j}=\left\{f_{l}^{i j} \in A_{i} r A_{j} \subseteq r \mid l=\right.$ $\left.1, \ldots, t_{i j}\right\}$ such that the image $\bar{X}_{i j}=\left\{\bar{f}_{l}^{i j} \mid l=1, \ldots, t_{i j}\right\}$ in ${ }_{i} M_{j}$ is a minimal generating set as an $A_{i}$ - $A_{j}$-bimodule. Let $X=\bigcup_{i, j \in \Delta_{0}} X_{i j}$. Then, its image $\bar{X}=\bigcup_{i, j \in \Delta_{0}} \bar{X}_{i j}$ generates $r / r^{2}=\sum_{i, j \in \Delta_{0}} A_{i}\left(r / r^{2}\right) A_{j}$ as an $A_{0}-A_{0}$-bimodule.

We now show that the subalgebra $S$ of $A$ generated by $A_{0}$ and the set $X$ is actually $A$. Consider the associated graded algebra $\operatorname{gr}(A)=\bigoplus_{s>0} r^{s} / r^{s+1}$, with $r^{0}=A$. Then $\operatorname{gr}(S)=\bigoplus_{s>0}\left(S \cap r^{s}\right) /\left(S \cap r^{s+1}\right)$ is the graded subalgebra of $\operatorname{gr}(A)$ generated by $A_{0}$ and $\bar{X}=\left\{\bar{f}_{l}^{i j} \mid 1 \leq l \leq t_{i j}, i, j \in \Delta_{0}\right\}$.

We claim that $\operatorname{gr}(S)=\operatorname{gr}(A)$. We will show that $S_{p}=r^{p} / r^{p+1}$, where $S_{p}=$ $\left(S \cap r^{p}\right) /\left(S \cap r^{p+1}\right)$ are the homogeneous components of the graded algebras gr $S$. Note that both $S_{p}$ and $r^{p} / r^{p+1}$ are $A_{0}-A_{0}$-bimodules. It follows from the definition that $S_{1}=\left(A_{0} X A_{0}\right) /\left(r^{2} \cap S\right) \subseteq A_{0} \bar{X} A_{0}=r / r^{2}$. By the choice of the set $\bar{X}$, we have that $A_{0} X A_{0}$ maps onto $r / r^{2}$ (under the quotient map $r \rightarrow r / r^{2}$ ). Hence, we have $S_{1}=r / r^{2}$. The multiplication

$$
\underbrace{r / r^{2} \otimes_{A_{0}} r / r^{2} \otimes_{A_{0}} \cdots \otimes_{A_{0}} r / r^{2}}_{p \text { times }} \rightarrow r^{p} / r^{p+1}
$$

in $\operatorname{gr}(A)$ is surjective following the definition of $r^{p}$. Thus

$$
r^{p} / r^{p+1}=\underbrace{S_{1} \cdots S_{1}}_{p \text { times }} \subseteq S_{p} .
$$

However, we have $S_{p} \subseteq r^{p} / r^{p+1}$. Hence $S_{p}=r^{p} / r^{p+1}$ and $\operatorname{gr}(A)=\operatorname{gr}(S)$.

We now prove that $S=A$. Otherwise, we must have $r \nsubseteq S$ since $A$ is generated by $A_{0}$ and $r$. Let $p$ be maximal such that $r^{p} \subseteq S$ is not true. Such a $p$ exists since 
$r^{m}=0$ for $m \gg 0\left(A\right.$ is Artinian) and $p \geq 1$. Take $a \in r^{p} \backslash S$ and let $\bar{a} \in r^{p} / r^{p+1}$ be the image of $a$. Since $S_{p}=r^{p} / r^{p+1}$, there is $b \in S \cap r^{p}$ such that $\bar{b}=\bar{a}$ in $r^{p} / r^{p+1}$. Thus $a-b \in r^{p+1} \subseteq S$ ( $p$ is maximal). Hence, $a=b+(a-b) \in S \cap r^{p}$. This is a contradiction. Hence we have $S=A$.

The fact that $A$ splits over $r$ and $A_{0}$ is a subalgebra of $A$ such that the quotient map $A \rightarrow A / r$ restricts to $A_{0}$ is the identity map. Then $A_{0} X A_{0} \subseteq A$ is an $A_{0}-A_{0}$-sub-bimodule of $A$. Since $|\Omega(i, j)|=t_{i j}=r k\left({ }_{i} M_{j}\right)$, there is a surjective homomorphism of $A_{0}$ - $A_{0}$-bimodules: $A_{0} \Omega(i, j) A_{0} \rightarrow A_{0} X_{i j} A_{0}$. Now by the universal mapping property of the generalized path algebra, there is a surjective algebra homomorphism $\phi: k\left(\Delta_{A}, \mathcal{A}\right) \rightarrow S$, since $S$ is generated by $A_{0}$ and the set $X$ as a subalgebra of $A$. Hence $\phi(k(\Delta, \mathcal{A}))=S=A$, as we have just proved, and $\left.\phi\right|_{A_{0}}=\operatorname{id}_{A_{0}}$.

Let $I=\operatorname{ker}(\phi)$ and $J$ be the ideal of $k\left(\Delta_{A}, \mathcal{A}\right)$ generated by all virtual $\mathcal{A}$-paths of length 1. Then $\phi(J)=r$ and thus induces $k(\Delta, \mathcal{A}) / J \cong A / r$. Hence $\operatorname{ker}(\phi) \subseteq J$. Since $\phi\left(J^{p}\right)=r^{p}$ for all $p \geq 0$, we have $\phi\left(J^{s}\right)=r^{s}=0$ for some $s>0$. This completes the proof of the theorem.

Theorem 3.4 requires that the Artinian algebra $A$ is splitting over its radical, i.e., the property (a) only without (b) as mentioned above, although the tensor algebra has to be replaced by the generalized path algebra. In the case $r_{A}^{2}=0$, condition (b) is automatic provided (a) holds.

For an Artinian algebra $A$, set $A_{0}=A / r_{A}$, which is a semi-simple algebra with an $A_{0}-A_{0}$-bimodule structure on $M=r_{A} / r_{A}^{2}$. Then we can call the ordered pair $\left(A_{0}, r_{A} / r_{A}^{2}\right)$ a generalized $k$-species in the language of $k$-species discussed in $[\mathrm{R}$. The tensor algebra $T_{A_{0}}(M)=\bigoplus_{n=0}^{\infty} M^{\otimes_{A_{0}} n}$ is an associate algebra (not necessarily Artinian). The generalized path algebra of a quiver is naturally the tensor algebra of a generalized $k$-species. The properties of this path algebra are controlled by the bi-module structure of $M$. This algebra plays an important role in non-commutative geometry, which will be studied in the next paper.

The following example shows the difference between the generalized path algebra and the tensor algebra of the associated generalized $k$-species in the fact that $\phi$ does not exist with condition (a) only.

One also notes that the surjective algebra homomorphism $\pi: k\left(\Delta_{A}, \mathcal{A}\right) \rightarrow$ $T_{A_{0}}\left(r_{A} / r_{A}^{2}\right)$ is an isomorphism if and only if $A_{i}\left(r_{A} / r_{A}^{2}\right) A_{j}$ is a free $A_{i} \otimes_{k} A_{j-}$ bimodule for all $i, j$. One natural question is, for a general $\pi$, whether there is a map $\psi: T_{A_{0}}\left(r_{A} / r_{A}^{2}\right) \rightarrow A$ such that $\psi \circ \pi=\phi$. The following example gives an answer to this question. It also shows that one cannot expect to generalize DK, Th. 8.5.2] to non-separable cases.

Example 3.5. Let $D=k[\alpha]$ with $k=\mathbb{F}_{p}(t)$ being the transcendental extension of the finite field with $p$ elements and $\alpha=\sqrt[p]{t}$. Then $D$ is a purely inseparable field extension of $k$. Now $B=D \otimes_{k} D$ is not semisimple, and its radical $r_{B}$ is nilpotent and generated by $x=\alpha \otimes_{k} 1-1 \otimes_{k} \alpha$ be a $D$-D-subbimodule of $B$. Let $A=D \oplus r_{B}$ be a $k$-vector space with multiplication defined by $(d, z)\left(d^{\prime}, z\right)=\left(d d^{\prime}, d z^{\prime}+z d^{\prime}+z z^{\prime}\right)$ with $d, d^{\prime} \in D$ and $z, z^{\prime} \in r_{B}$. Here $d d^{\prime}$ and $z z^{\prime}$ are the multiplications in $D$ and $r_{B}$ respectively by noting the $D$ - $D$-bimodule structure on $r_{B}$. This makes $A$ an associative algebra and $A / r_{A}=D$ is not separable. However, $A$ is splitting over its radical. $A$ is a not a commutative algebra (unless $p=2$ ) and $r_{A} / r_{A}^{2}=r_{B} / r_{B}^{2} \cong D$ are $D$-D-bimodules whose actions are coinciding with the left and right actions 
of $D$. Note that the left and right actions of $D$ on $r_{B}$ are not the same unless $p=2$. Hence the tensor algebra $T_{D}\left(r_{A} / r_{A}^{2}\right)$ is a commutative algebra, but $A$ is not commutative. Hence there is no surjective $k$-algebra map $\psi: T_{D}\left(r_{A} / r_{A}^{2}\right) \rightarrow A$.

This example shows that under the assumption that $A$ is splitting over its radical, the surjective map $\phi: k\left(\Delta_{A}, \mathcal{A}\right) \rightarrow A$ in Theorem 3.4 cannot be factored through $T_{A_{0}}\left(r_{A} / r_{A}^{2}\right)$.

As mentioned earlier the separability condition on $A_{0}$ implies that $A$ is splitting over its radical, by the Wedderburn-Malcev theorem. There have been numerous generalizations of the Wedderburn-Malcev theorem in the literature. Combining Theorem 3.3 and Theorem 3.4, the following gives a characterization of Artinian algebras that is splitting over its radical in terms of natural quivers and the associated generalized path algebras.

Corollary 3.6. An Artinian k-algebra $A$ is splitting over its radical if and only if $A$ is of Gabriel type for generalized path algebras (cf. Definition 3.2); i.e., there is a surjective algebra homomorphism $\pi: k\left(\Delta_{A}, \mathcal{A}\right) \rightarrow A$ such that $\operatorname{ker}(\pi) \subseteq J$.

We will see in Section 6 that it will be important if the condition $\operatorname{ker}(\pi) \subset J$ in Corollary 3.6 is replaced by the stronger one, that is, $\operatorname{ker}(\pi) \subset J^{2}$. In this case, the ideal $I=\operatorname{ker}(\pi)$ is said to be admissible.

\section{The Relations among quivers arisen from an Artinian algebra TO ITS GENERALIZED PATH ALGEBRA}

In this section, we use the relation between the Ext-quiver and the natural quiver in Section 2 to study the associated normal generalized path algebras of Artinian algebras. Note that the definition of the Ext-quiver always requires that an Artinian algebra $A$ is splitting over the field $k$, which we will assume in this section.

The natural quiver $\Delta_{A}$ of an Artinian algebra $A$ is always finite, i.e., including finitely many vertices and finitely many arrows. In the sequel, we always assume that $\Delta_{A}$ is acyclic (i.e., $\Delta_{A}$ does not have oriented cycles of length at least 1 ). Trivially, this is the sufficient and necessary condition under which the associated $k\left(\Delta_{A}, \mathcal{A}\right)$ is Artinian.

By definition, acyclicity of $\Delta_{A}$ implies that the natural quiver of $k\left(\Delta_{A}, \mathcal{A}\right)$ is just that of $A$, that is, $\Delta_{k\left(\Delta_{A}, \mathcal{A}\right)}=\Delta_{A}$. Denote by $m_{i j}$ and $g_{i j}$, respectively, the arrow multiplicities in the Ext-quivers $\Gamma_{A}$ and $\Gamma_{k\left(\Delta_{A}, \mathcal{A}\right)}$, respectively. Note that they should not be confused with each other. In general, $m_{i j}$ and $g_{i j}$ are quite different since the representation theories of $A$ and $k\left(\Delta_{A}, \mathcal{A}\right)$ are quite different (cf. Section 5).

For the radical $J$ of $k\left(\Delta_{A}, \mathcal{A}\right), k\left(\Delta_{A}, \mathcal{A}\right) / J \cong A / r_{A}=\bigoplus_{i \in \Delta_{0}} A_{i}$. By Theorem 2.2 , the natural quiver $\Delta_{k\left(\Delta_{A}, \mathcal{A}\right)}$ is a sub-quiver of the Ext-quiver $\Gamma_{k\left(\Delta_{A}, \mathcal{A}\right)}$, and exactly,

$$
t_{i j}=\left\lceil\frac{g_{i j}}{n_{i} n_{j}}\right\rceil
$$

Denote $I=\left(\Delta_{A}\right)_{0}$. A complete set of non-isomorphic primitive orthogonal idempotents of $A$ is a set of primitive orthogonal idempotents $\left\{e_{i}: i \in I\right\}$ such that $A e_{i} \neq A e_{j}$ as left $A$-modules for any $i \neq j$ in $I$ and for each primitive idempotent $e$ the module $A e$ is isomorphic to one of the modules $A e_{i}(i \in I)$.

Let $\bar{e}_{i}=e_{i}+r_{A}$. Then, by [L], $\left\{\bar{e}_{i}: i \in I\right\}$ is a complete set of non-isomorphic primitive orthogonal idempotents of $A / r$. Let $\tilde{e}_{i} \in k\left(\Delta_{A}, \mathcal{A}\right.$ ) be the lift of $\bar{e}_{i}$ (we 
have assumed that $k\left(\Delta_{A}, \mathcal{A}\right)$ is Artinian). Then, $S_{i} \cong A e_{i} / r_{A} e_{i}=\left(A / r_{A}\right) \bar{e}_{i} \cong$ $\left(k\left(\Delta_{A}, \mathcal{A}\right) \tilde{e}_{i}\right) /\left(J \tilde{e}_{i}\right)$, as $i \in I$, gives a list of all non-isomorphic irreducible modules for both $A$ and $k\left(\Delta_{A}, \mathcal{A}\right)$.

For $i \in I$, the identity $1_{A_{i}}$ of $A_{i}$ can be decomposed into a sum of primitive idempotents, i.e., $1_{A_{i}}=e_{11}^{i}+\cdots+e_{n_{i} n_{i}}^{i}$, and we can assume $e_{11}^{i}=e_{i}$.

Note that $A$ is $k$-splitting. By [ARS], Proposition III.1.14, we have

$$
g_{i j}=\operatorname{dim}_{k} \operatorname{Ext}_{k\left(\Delta_{A}, \mathcal{A}\right)}^{1}\left(S_{i}, S_{j}\right)=\operatorname{dim}_{k}\left(\tilde{e}_{k k}^{j} J / J^{2} \tilde{e}_{l l}^{i}\right)
$$

for all $k, l$. Therefore,

$$
\begin{aligned}
\operatorname{dim}_{k} A_{j} J / J^{2} A_{i} & =\operatorname{dim}_{k} 1_{A_{j}} J / J^{2} 1_{A_{i}}=\sum_{k=1}^{n_{j}} \sum_{l=1}^{n_{i}} \operatorname{dim}_{k} e_{k k}^{j} J / J^{2} e_{l l}^{i} \\
& =n_{i} n_{j} \operatorname{dim}_{k} \operatorname{Ext}_{k\left(\Delta_{A}, \mathcal{A}\right)}^{1}\left(S_{i}, S_{j}\right)=n_{i} n_{j} g_{i j} .
\end{aligned}
$$

Recall that the number of arrows from $i$ to $j$ in $\Delta_{k\left(\Delta_{A}, \mathcal{A}\right)}=\Delta_{A}$ is $t_{i j}$. These $t_{i j}$ arrows generate freely the $A_{j}-A_{i}$-bimodule $A_{j} J / J^{2} A_{i}$. Thus,

$$
\operatorname{dim}_{k} A_{j} J / J^{2} A_{i}=\operatorname{dim}_{k} A_{j} \operatorname{dim}_{k}\left(J / J^{2}\right) \operatorname{dim}_{k} A_{i}=n_{j}^{2} t_{i j} n_{i}^{2} .
$$

Hence, $g_{i j}=n_{i} n_{j} t_{i j}$, that is, we obtain the following:

Proposition 4.1. Let $A$ be a $k$-splitting finite-dimensional algebra with radical $r$, whose natural quiver $\Delta_{A}$ is acyclic. Write $A / r=\bigoplus_{i \in\left(\Delta_{A}\right)_{0}} A_{i}$, where $A_{i}$ are simple algebra for all $i$ with $n_{i}=\sqrt{\operatorname{dim}_{k} A_{i}}$. Then, the natural quiver of $k\left(\Delta_{A}, \mathcal{A}\right)$ is the same as that of $A$ and

$$
g_{i j}=n_{i} n_{j} t_{i j}
$$

where $g_{i j}$ is the number of arrows from $i$ to $j$ in $\Gamma_{k\left(\Delta_{A}, \mathcal{A}\right)}$ and $t_{i j}$ is the number of arrows from $i$ to $j$ in $\Delta_{A}$.

For the number $m_{i j}$ of arrows from $i$ to $j$ in $\Gamma_{A}$, by Theorem 2.2, $t_{i j}=\left\lceil\frac{m_{i j}}{n_{i} n_{j}}\right\rceil$. Then, $\left\lceil\frac{m_{i j}}{n_{i} n_{j}}\right\rceil=\frac{g_{i j}}{n_{i} n_{j}}$. Equivalently, we have

Corollary 4.2. $m_{i j} \leq g_{i j}<m_{i j}+n_{i} n_{j}$.

The set $\left\{P_{i}=A e_{i} \mid i \in I\right\}$ is a complete set of representatives of the iso-class of indecomposable projective $A$-modules. Then the basic algebra $B$ of $A$ is given by

$$
B=\operatorname{End}_{A}\left(\coprod_{i \in I} P_{i}\right) \cong \bigoplus_{i, j \in I} \operatorname{Hom}_{A}\left(P_{i}, P_{j}\right) \cong \bigoplus_{i, j \in I} e_{i} A e_{j} .
$$

Similarly the basic algebra of $k\left(\Delta_{A}, \mathcal{A}\right)$ is

$$
C=\operatorname{End}_{k\left(\Delta_{A}, \mathcal{A}\right)}\left(\bigoplus_{i \in I} k\left(\Delta_{A}, \mathcal{A}\right) \bar{e}_{i}\right) \cong \bigoplus_{i, j \in I} \bar{e}_{i} k\left(\Delta_{A}, \mathcal{A}\right) \bar{e}_{j} .
$$

The relationship between the two basic algebras $B$ and $C$ is given in $[\mathrm{LC}]$ when $A$ is of Gabriel type.

As we have said, $k\left(\Delta_{A}, \mathcal{A}\right)$ is not Artinian when $\Delta_{A}$ has an oriented cycle. Hence, we cannot affirm whether $C$ is Morita equivalent to $k\left(\Delta_{A}, \mathcal{A}\right)$ in general. However, $C$ is still decided uniquely by $k\left(\Delta_{A}, \mathcal{A}\right)$. Therefore, we call $C$ the basic algebra associated to $k\left(\Delta_{A}, \mathcal{A}\right)$.

As we have assumed $\Delta_{A}$ is acyclic in this section, $C$ is Morita equivalent to $k\left(\Delta_{A}, \mathcal{A}\right)$ since $k\left(\Delta_{A}, \mathcal{A}\right)$ is Artinian. Hence, their Ext-quivers are the same, that is, $\Gamma_{k\left(\Delta_{A}, \mathcal{A}\right)}=\Gamma_{C}$. Also, since $C$ is basic, $\Gamma_{C}=\Delta_{C}$. 
To sum up, assuming that $\Delta_{A}$ is acyclic, we get the following diagram:

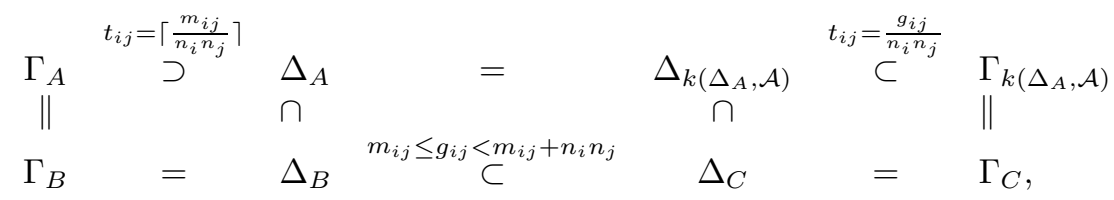

where $C, \supset$ and $\cap$ mean the embeddings of the dense sub-quivers.

\section{DiAgRAM FOR NON-SPLITTING ALGEBRAS}

In Sections 2 and 4 , the Artinian algebra $A$ is required to be splitting over the ground field $k$ due to the definition of the Ext-quiver. If $A$ is not splitting over $k$, usually the Ext-quiver and its representations have to be respectively replaced by the so-called valued quiver or $k$-species. On the other hand, the notion of the diagram of an Artinian algebra is introduced in [DK] in the case when $A$ is not necessarily splitting over $k$. Now, we recall the definition of the diagram of an Artinian algebra $A$.

Let $P_{1}, P_{2}, \cdots, P_{s}$ be pairwise non-isomorphic principal indecomposable projective modules over an Artinian algebra $A$, corresponding to the simple components $A_{1}, A_{2}, \cdots, A_{s}$ of the semisimple algebra $\bar{A}=A / r=\bigoplus_{i=1}^{s} A_{i}$. Write $R_{i}=r P_{i}$; then $S_{i}=P_{i} / R_{i}$ is the corresponding irreducible $A$-module. At the same time, $V_{i}=R_{i} / r R_{i}$ is a semisimple left $A$-module which has a direct sum decomposition $V_{i} \cong \bigoplus_{j=1}^{s} S_{j}^{\oplus h_{i j}}$ for some unique integers $h_{i j}$. Define the quiver $D_{A}=\left(D_{0}, D_{1}\right)$ by setting $D_{0}=\{1, \cdots, s\}$ and the arrow set $D_{1}$ such that there are exactly $h_{i j}$ many arrows from $i$ to $j$ for $i, j \in D_{0}$. This quiver $D_{A}$ is called the diagram of the algebra $A$.

Observe that: (i) the projective cover of $V_{i}$ is $P\left(R_{i}\right) \cong \bigoplus_{j=1}^{s} P_{j}^{\oplus h_{i j}}$ for each $i$; (ii) two Morita equivalent algebras have the same diagrams; (iii) the diagrams of $A$ and $A / r^{2}$ coincide.

Proposition 5.1. Let $S_{1}, S_{2}, \cdots, S_{s}$ be pairwise non-isomorphic irreducible modules over an Artinian algebra $A$. Then, in the diagram $D_{A}$ of $A$, the number of arrows from the vertex $i$ to the vertex $j$ is

$$
h_{i j}=\operatorname{dim}_{D_{j}} \operatorname{Ext}_{A}\left(S_{i}, S_{j}\right),
$$

where $D_{j}=\operatorname{End}_{A}\left(S_{j}\right)$ is a division algebra.

Proof. Applying the functor $\operatorname{Hom}_{A}\left(-, S_{j}\right)$ to the short exact sequence $0 \rightarrow R_{i} \rightarrow$ $P_{i} \rightarrow S_{i} \rightarrow 0$, one gets the long exact sequence, which gives the isomorphisms

$$
\operatorname{Ext}_{A}^{p}\left(R_{i}, S_{j}\right) \cong \operatorname{Ext}_{A}^{p+1}\left(S_{i}, S_{j}\right) \quad(p \geq 0) .
$$

Since $R_{i} / r R_{i}=\bigoplus_{l=1}^{s} S_{l}^{\oplus h_{i l}}$, we have $\operatorname{Hom}_{A}\left(R_{i} / r R_{i}, S_{j}\right) \cong D_{j}^{h_{i j}}$. Now the proposition follows from $\operatorname{Hom}_{A}\left(R_{i} / r R_{i}, S_{j}\right) \cong \operatorname{Hom}_{A}\left(R_{i}, S_{j}\right)$.

When $A$ is $k$-splitting, each $D_{j}=k$. Then $h_{i j}=\operatorname{dim}_{k} \operatorname{Ext}_{A}^{1}\left(S_{i}, S_{j}\right)=m_{i j}$. Hence, in this case, the Ext-quiver $\Gamma_{A}$ is equal to the diagram $D_{A}$, whose relationship with $\Delta_{A}$ is given in Theorem 2.2. When $A$ is not $k$-splitting, in place of the Ext-quiver $\Gamma_{A}$ is a valued quiver with modulation. The following gives a relation of the diagram $D_{A}$ with the valued quiver and associated modulation as well as with the natural quiver $\Delta_{A}$ for a general Artinian algebra $A$. 
Using the earlier notation, $A / r=A_{1} \oplus \cdots \oplus A_{s}$ with $A_{i} \cong M_{n_{i}}\left(D_{i}\right)$ (the matrix algebra with entries in $D_{i}$ ). Let $e_{p q}^{i}$ be the matrix basis element of $A_{i}$ with 1 at the $(p, q)$ position and zero anywhere else. The irreducible left $A_{i}$-module is $S_{i} \cong A_{i} e_{11}^{i}$ and irreducible right $A_{i}$-module is $S_{i}^{o p} \cong e_{11}^{i} A_{i}$. The $A_{j}$ - $A_{i}$-module $A_{j}\left(r / r^{2}\right) A_{i}$ is semisimple both as a left $A_{j}$-module and as a right $A_{i}$-module. Let $D_{l}$ act on $S_{l}$ from right and $D_{l}$ act on $S_{l}^{o p}$ from left. For any $A_{j}-A_{i}$-bi-module $M$ which is semisimple as an $A_{j}$-module and an $A_{i}$-module respectively, we have the following:

(a) $\operatorname{Hom}_{A_{j}}\left(S_{j}, M\right)$ is a $D_{j}$ - $A_{i}$-bi-module isomorphic to $e_{11}^{j} M$, and the map $S_{j} \otimes_{D_{j}} \operatorname{Hom}_{A_{j}}\left(S_{j}, M\right) \rightarrow M$ defined by $x \otimes \phi=\phi(x)$ is an isomorphism of $A_{j}$ - $A_{i}$-bi-modules;

(b) $\operatorname{Hom}_{A_{i}}\left(S_{i}^{o p}, \operatorname{Hom}_{A_{j}}\left(S_{j}, M\right)\right)$ is a $D_{j}$ - $D_{i}$-bimodule isomorphic to $e_{11}^{j} M e_{11}^{i}$, and the map $\operatorname{Hom}_{A_{i}}\left(S_{i}^{o p}, \operatorname{Hom}_{A_{j}}\left(S_{j}, M\right)\right) \otimes_{D_{i}} S_{i}^{o p} \rightarrow \operatorname{Hom}_{A_{j}}\left(S_{j}, M\right)$ defined by $f \otimes v=f(v)$ is an isomorphism of an $D_{j}$ - $A_{i}$-bi-modules.

(c) The natural multiplication map $S_{j} \otimes_{D_{j}} e_{11}^{j} M e_{11}^{i} \otimes S_{i}^{o p} \rightarrow M$ is an isomorphism of an $A_{j}$ - $A_{i}$-bi-module.

Taking $M=A_{j}\left(r / r^{2}\right) A_{i}$, we have an $A_{j}$ - $A_{i}$-bi-module isomorphism

$$
A_{j}\left(r / r^{2}\right) A_{i} \cong S_{j} \otimes_{D_{j}} e_{11}^{j}\left(r / r^{2}\right) e_{11}^{i} \otimes_{D_{i}} S_{i}^{o p} .
$$

Denote by $\operatorname{Arrow}_{\Delta_{A}}(i, j)$ the $k$-linear space generated by the set of arrows from $i$ to $j$ in the natural quiver $\Delta_{A}$. Then, $t_{i j}=\operatorname{dim}_{k} \operatorname{Arrow}_{\Delta_{A}}(i, j)$ is the minimal number of generators of $A_{j}\left(r / r^{2}\right) A_{i}$ as an $A_{j}$ - $A_{i}$-module. Therefore there is a surjective homomorphism of $A_{j}$ - $A_{i}$-bimodules

$$
A_{j} \otimes_{k} \operatorname{Arrow}_{\Delta_{A}}(i, j) \otimes_{k} A_{i} \rightarrow A_{j}\left(r / r^{2}\right) A_{i} .
$$

In particular, by taking $M=A_{j}\left(r / r^{2}\right) A_{i}$, we have a surjective $A_{j}$ - $A_{i}$-bimodule homomorphism

$$
A_{j} \otimes_{k} \operatorname{Arrow}_{\Delta_{A}}(i, j) \otimes_{k} A_{i} \rightarrow S_{j} \otimes_{D_{j}} e_{11}^{j}\left(r / r^{2}\right) e_{11}^{i} \otimes_{D_{i}} S_{i}^{o p} .
$$

Since $A_{i} \cong S_{i} \otimes_{D_{i}} S_{i}^{o p}$ as an $A_{i}$ - $A_{i}$-bimodule, by applying the exactor functors $\operatorname{Hom}_{A_{j}}\left(S_{j},-\right)$ and $\operatorname{Hom}_{A_{i}}\left(S_{i}^{o p},-\right)$ consecutively, we get a surjective map of $D_{j^{-}} D_{i^{-}}$ bimodules

$$
S_{j}^{o p} \otimes_{k} \operatorname{Arrow}_{\Delta_{A}}(i, j) \otimes_{k} S_{i} \rightarrow e_{11}^{j}\left(r / r^{2}\right) e_{11}^{i} .
$$

In the definition of the diagram $D_{A}$ of $A$, we have $V_{i} \cong\left(r / r^{2}\right) e_{11}^{i}$ as left $A$ modules. Then by (a) and (b) above, $h_{i j}=\operatorname{dim}_{D_{j}}\left(e_{11}^{j}\left(r / r^{2}\right) e_{11}^{i}\right)$. Note that $S_{j} \otimes_{D_{j}} e_{11}^{j}\left(r / r^{2}\right) e_{11}^{i} \otimes_{D_{i}} S_{i}^{o p}$ can be generated by $h_{i j}$ many generators as an $A_{j^{-}} A_{i^{-}}$ bimodule. Then (4) implies that $t_{i j} \leq h_{i j}$.

Since $A$ is Artinian, we can compare the $D_{j}$-dimensions from (5) to get

$$
h_{i j} \leq n_{i} n_{j} \operatorname{dim}_{k}\left(D_{i}\right) t_{i j} .
$$

In conclusion, we have

Lemma 5.2. For an Artinian k-algebra A, we have

$$
t_{i j} \leq h_{i j} \leq\left(n_{i} n_{j} \operatorname{dim}_{k} D_{i}\right) t_{i j} .
$$

Similar to the setup in Section 4 , let $\varepsilon_{i} \in A$ be idempotents that are inverse images of $e_{11}^{i}$ such that $\left\{\varepsilon_{1}, \cdots, \varepsilon_{s}\right\}$ is a complete set of non-isomorphic primitive idempotents of $A$. Set $\varepsilon=\sum_{i=1}^{s} \varepsilon_{i}$. Then $B=\operatorname{End}_{A}(A \varepsilon, A \varepsilon) \cong \varepsilon A \varepsilon$ is the basic algebra of $A$ with radical $r_{B}=\varepsilon r_{A} \varepsilon$. We know that the diagram $D_{B}$ of 
$B$ is the same as that of $A$. In this case $h_{i j}=\operatorname{dim}_{D_{j}} \varepsilon_{j} r_{B} / r_{B}^{2} \varepsilon_{i}$. The natural quiver $\Delta_{B}$ of $B$ has the number of arrows $\mid$ Arrow $_{\Delta_{B}}(i, j) \mid$ from $i$ to $j$ equal to the minimal number of generators of $\varepsilon_{j} r_{B} / r_{B}^{2} \varepsilon_{i}$ as $D_{j}$ - $D_{i}$-bimodules. Note that $\varepsilon_{j} r_{B} / r_{B}^{2} \varepsilon_{i} \cong e_{11}^{j} r_{A} / r_{A}^{2} e_{11}^{i}$ as $D_{j}$ - $D_{i}$-bimodules. Then $S_{j} \otimes_{D_{j}} e_{11}^{j} r_{A} / r_{A}^{2} e_{11}^{2} \otimes_{D_{i}} S_{i}^{o p}$ can be generated by $\mid$ Arrow $_{\Delta_{B}}(i, j) \mid$ many elements as an $A_{j}$ - $A_{i}$-bimodule. We therefore get

$$
\left|\operatorname{Arrow}_{\Delta_{A}}(i, j)\right| \leq\left|\operatorname{Arrow}_{\Delta_{B}}(i, j)\right| .
$$

For the basic algebra $B$, the system $\left\{\varepsilon_{j} r_{B} / r_{B}^{2} \varepsilon_{i} \mid i, j=1, \cdots, s\right\}$, together with $\left\{D_{i} \mid i=1, \cdots, s\right\}$, defines a $k$-species [R]. If all $D_{i}$ are finite dimensional over $k$, then the system is a modulation of the valued quiver for the algebra $A[\mathrm{DR}$.

Theorem 5.3. For an Artinian algebra $A$ with radical $r_{A}$, let $\left\{S_{1}, \cdots, S_{s}\right\}$ be the complete set of all non-isomorphic irreducible A-modules and $D_{i}=\operatorname{End}_{A}\left(S_{i}\right)$ for any $i=1, \cdots, s$. Let $B$ be the basic algebra of $A$ with radical $r_{B}$ such that $B / r_{B}=\prod_{i=1}^{s} D_{i}$. Let $t_{i j}^{A}$ (resp. $t_{i j}^{B}$ ) and $h_{i j}^{A}$ (resp. $h_{i j}^{B}$ ) be the number of arrows from $i$ to $j$ in the natural quiver $\Delta_{A}$ (resp. $\Delta_{B}$ ) and the diagram $D_{A}$ (resp. $D_{B}$ ) respectively. For any $i, j=1, \cdots, s$, we have

(i) $t_{i j}^{A} \leq\left\lceil\frac{t_{i j}^{B}}{n_{i} n_{j}}\right\rceil \leq t_{i j}^{B}$;

(ii) $t_{i j}^{A} \leq\left\lceil\frac{h_{i j}^{A}}{n_{i} n_{j}}\right\rceil \leq t_{i j}^{A} \operatorname{dim}_{k} D_{i}$.

Proof. It follows from Proposition 5.1 that $h_{i j}^{A}=h_{i j}^{B}$. If $M$ is a $D_{j}-D_{i}$-bimodule which can be generated by $m$ many elements as a $D_{j}$ - $D_{i}$-bimodule, then there is a surjective map $\left(D_{j} \otimes_{k} D_{i}\right)^{\oplus m} \rightarrow M$ as $D_{j}$ - $D_{i}$-bimodules. Thus we have a surjective map $S_{j} \otimes_{D_{j}}\left(D_{j} \otimes_{k} D_{i}\right)^{\oplus m} \otimes_{D_{i}} S_{i}^{o p} \rightarrow S_{j} \otimes_{D_{j}} M \otimes_{D_{i}} S_{i}^{o p}$ of $A_{j}$ - $A_{i}$-bimodules. Let $q=\left\lceil\frac{m}{n_{j} n_{i}}\right\rceil$. Hence we have a surjective map $\left(S_{j} \otimes_{k} S_{i}^{o p}\right)^{\oplus q n_{j} n_{i}} \rightarrow\left(S_{j} \otimes_{k} S_{i}^{o p}\right)^{\oplus m} \cong$ $S_{j} \otimes_{D_{j}}\left(D_{j} \otimes_{k} D_{i}\right)^{\oplus m} \otimes_{D_{i}} S_{i}^{o p}$ as $A_{j}$ - $A_{i}$-bimodules. Since $\left(S_{j} \otimes_{k} S_{i}^{o p}\right)^{\oplus n_{j} n_{i}} \cong$ $S_{j}^{\oplus n_{j}} \otimes_{k}\left(S_{i}^{o p}\right)^{\oplus n_{i}}$ as $A_{j}-A_{i}$-bimodules, which can be generated by one element as an $A_{j}$ - $A_{i}$-bimodule, $\left(S_{j} \otimes_{k} S_{i}^{o p}\right)^{\oplus q n_{j} n_{i}}$ can be generated by $q$ elements as an $A_{j}$ $A_{i}$-bimodule. Therefore $S_{j} \otimes_{D_{j}} M \otimes_{D_{i}} S_{i}^{o p}$ can be generated by $q$ elements as an $A_{j}$ - $A_{i}$-module.

Applying the above argument to $M=e_{11}^{j}\left(r_{A} / r_{A}^{2}\right) e_{11}^{i}$, where $M$ can be generated by $t_{i j}^{B}$ many elements as a $D_{j}$ - $D_{i}$-bimodule, we conclude that $A_{j}\left(r_{A} / r_{A}^{2}\right) A_{i}$ can be generated by $\left\lceil\frac{t_{i j}^{B}}{n_{j} n_{i}}\right\rceil$ many elements as $A_{j}-A_{i}$-bimodules. This shows that $t_{i j}^{A} \leq$ $\left\lceil\frac{t_{i j}^{B}}{n_{j} n_{i}}\right\rceil \leq t_{i j}^{B}$, where the first inequality follows from the fact that $t_{i j}^{A}$ is the minimum.

To show (ii), we note that $t_{i j}^{B} \leq h_{i j}^{B}=h_{i j}^{A} \leq n_{i} n_{j} \operatorname{dim}_{k} D_{i} t_{i j}^{A}$ by Lemma [5.2, By (i), we have $t_{i j}^{A} \leq\left\lceil\frac{t_{i j}^{B}}{n_{i} n_{j}}\right\rceil \leq\left\lceil\frac{h_{i j}^{A}}{n_{i} n_{j}}\right\rceil \leq\left\lceil\frac{n_{i} n_{j} \operatorname{dim}_{k} D_{i} t_{i j}^{A}}{n_{i} n_{j}}\right\rceil=t_{i j}^{A} \operatorname{dim}_{k} D_{i}$.

The relation (ii) implies that $t_{i j}$ is never larger than $h_{i j}$, and $t_{i j} \neq 0$ if and only if $h_{i j} \neq 0$ for any $i, j \in\left(\Delta_{A}\right)_{0}$, which gives the relation between the natural quiver and the diagram.

The relation (i) gives the relation between the natural quiver and the Ext-quiver of an Artinian algebra over an arbitrary field $k$. Note that the formula $t_{i j}=\left\lceil\frac{m_{i j}}{n_{i} n_{j}}\right\rceil$ in Theorem 2.2 holds only in the case that $A$ is $k$-splitting, since the diagram $D_{A}$ and the ext-quiver $\Gamma_{A}$ are the same when $A$ is splitting over $k$. 
In general, when $A$ is basic (i.e, $A_{i}=D_{i}$ is a division ring) without the splitting condition over $k$, the natural quiver $\Delta_{A}$ will not be the same as either $\Gamma_{A}$ or $D_{A}$. In this case, the discussion before Theorem 5.3 relates $\Delta_{A}$ and $D_{A}$ by the species $\left\{D_{i}, \varepsilon_{j} r_{A} / r_{A}^{2} \varepsilon_{i}\right\}[\mathrm{R}]$ as follows:

(i) $h_{i j}=\operatorname{dim}_{D_{j}}\left(A_{j} r_{A} / r_{A}^{2} A_{i}\right)$ and $t_{i j}$ is a minimal number of generators of $A_{j} r_{A} / r_{A}^{2} A_{i}$ as an $A_{j}$ - $A_{i}$-bimodule;

(ii) if in addition $A$ is splitting over $k$, then $h_{i j}=t_{i j}=m_{i j}$, which means $D_{A}=\Delta_{A}=\Gamma_{A}$.

Example 5.4. Let $E / k$ be a field extension. Consider the algebra $A=\left[\begin{array}{cc}E & M \\ 0 & E\end{array}\right]$ as a basic algebra over $k$ for any $E$-E-bimodule $M$. With different choices of $M$ one can have either $h_{i j}=t_{i j}$ (taking $M=E$ ) or $h_{i j}$ and $t_{i j}$ to be quite different (taking $M=E \otimes_{k} E$ ).

\section{Hereditary algebras as Generalized Path AlgEbras}

We have mentioned the result in DK that any finite-dimensional hereditary algebra $A$ is isomorphic to the tensor algebra $T\left(A / r, r / r^{2}\right)$ if the quotient algebra $A / r$ is separable for the radical $r$. As a comparison, in this section we will show that an Artinian hereditary algebra $A$ is always isomorphic to $k\left(\Delta_{A}, \mathcal{A}\right)$ if $A$ is of Gabriel type with an admissible defining ideal.

It is proved in $\left[\mathrm{DK}\right.$, Corollary 3.7.3, that the diagram $D_{A}$ has no cycles if $A$ is a finite-dimensional hereditary algebra. In fact this is true for Artinian hereditary algebras.

Lemma 6.1 ([HGK]). If a ring $A$ is hereditary, then any non-zero homomorphism $\varphi: P_{1} \rightarrow P_{2}$ of indecomposable projective A-modules is a monomorphism.

Lemma 6.2. If an Artinian algebra $A$ is hereditary, then its diagram $D_{A}$ has no cycles.

Proof. For any vertices $i$ and $j$, if there is an arrow $\sigma$ from $j$ to $i$ in $D_{A}$, then there exists a non-zero homomorphism $f_{\sigma}: P_{j} \rightarrow P_{i}$ of projective covers of irreducible modules $S_{j}$ and $S_{i}$ such that $f_{\sigma}\left(P_{j}\right) \subseteq r P_{i}$. By Lemma 6.1, $f_{\sigma}$ is always a monomorphism, but not onto.

Suppose $D_{A}$ has a cycle $\sigma_{1} \sigma_{2} \cdots \sigma_{s}$ with both tail and head at the vertex $i$. Then, $f=f_{\sigma_{1}} f_{\sigma_{2}} \cdots f_{\sigma_{s}}$ is a monomorphism from $P_{i}$ to $P_{i}$ since each $f_{\sigma_{i}}$ is a monomorphism for any $i$. It is not isomorphic, i.e. $f\left(P_{i}\right) \varsubsetneqq P_{i}$. Moreover, it follows that the infinite sequence

$$
P_{i} \supseteqq f\left(P_{i}\right) \supseteqq f^{2}\left(P_{i}\right) \supseteqq \cdots \supseteqq f^{l}\left(P_{i}\right) \supseteqq \cdots .
$$

Note that $P_{i}$ is isomorphic to a left ideal of $A$. Thus the above contradicts the fact that $A$ is Artinian.

Proposition 6.3. Let $A$ be a hereditary Artinian algebra. Then the natural quiver $\Delta_{A}$ of $A$ is finite and acyclic.

Proof. Finiteness of $\Delta_{A}$ is trivial. According to the relation between $\Delta_{A}$ and $D_{A}$ in Theorem 5.3, $\Delta_{A}$ is acyclic if and only if $D_{A}$ is acyclic. By Lemma 6.2, $D_{A}$ is acyclic. Hence, $\Delta_{A}$ is acyclic. 
By definition, normal generalized path algebras can be thought of as a special class of tensor algebras, which are always hereditary due to [ARS]. The following main result in this section can be thought of as a partial converse of this statement.

Proposition 6.4. Let $\pi: B \rightarrow A$ be a surjective homomorphism of two hereditary algebras $A$ and $B$ such that $I=\operatorname{ker}(\pi) \subseteq \operatorname{rad}(B)^{2}$. If $B$ is Artinian, then $I=0$ and $\pi$ is an isomorphism.

Proof. Let $r_{B}=\operatorname{rad}(B)$. Since $B$ is Artinian, $r_{B}^{n}=0$ for some $n$. We have $A \cong B / I$ and $r_{B}^{s} \subset I \subset r_{B}^{2}$ for some positive integer $s$. It is enough to prove that $I=0$.

Let $R=r_{B} / I$. By induction on $k$, we will prove that for any $k \geq 0$,

$$
R^{k} / R^{k+1} \cong r_{B}^{k} / r_{B}^{k+1}
$$

as $A$-modules. Here the $A$-module structure of $r_{B}^{k} / r_{B}^{k+1}$ can be induced naturally from the $B$-module structure of $r_{B}^{k} / r_{B}^{k+1}$ since $I\left(r_{B}^{k} / r_{B}^{k+1}\right)=0$.

When $k=1$, we have $R / R^{2}=\left(r_{B} / I\right) /\left(r_{B} / I\right)^{2}=\left(r_{B} / I\right) /\left(r_{B}^{2} / I\right) \cong r_{B} / r_{B}^{2}$ since $I \subset r_{B}^{2}$.

Suppose that (7) holds for $k-1 \geq 0$, that is, $R^{k-1} / R^{k} \cong r_{B}^{k-1} / r_{B}^{k}$ as $A$-modules. For the case of $k$, we discuss it as follows.

We first note that for any projective $B$-module $Q, Q / I Q=A \otimes_{B} Q$ is a projective $A$-module. In particular, for any $A$-module $M$, if $P_{B}(M)$ is the projective cover of $M$ as a $B$-module, then $A \otimes_{B} P_{B}(M)$ is the projective cover of $M / I M$ as an $A$-module. Now let $\bar{P}=P_{A}\left(R^{k-1} / R^{k}\right)$ be the projective covers of $R^{k-1} / R^{k}$ as $A$-modules, and let $Q=P_{B}\left(r_{B}^{k-1} / r_{B}^{k}\right)$ be the projective cover of $r_{B}^{k-1} / r_{B}^{k}$ as a $B$-module. Thus by induction assumption, $r_{B}^{k-1} / r_{B}^{k} \cong R^{k-1} / R^{k}$ as an $A$-module. Then we have $\bar{P} \cong Q / I Q$ as $A$-modules (by [Lam], pp. 363-364). Letting $\pi: Q \rightarrow$ $Q / I Q$ be the quotient map, we have

$$
\begin{aligned}
R \bar{P} \cong \pi\left(r_{B}\right) \pi(Q) & =\pi\left(r_{B} Q\right) \cong r_{B} Q / I Q \\
R^{2} \bar{P} \cong \pi\left(r_{B}^{2}\right) \pi(Q) & =\pi\left(r_{B}^{2} Q\right) \cong r_{B}^{2} Q / I Q
\end{aligned}
$$

Since $R=r_{B} / I \cong \operatorname{rad} A$, we have $R^{k}=R R^{k-1} \cong(\operatorname{rad} A) R^{k-1}=\operatorname{rad} R^{k-1}$; thus $\bar{P}=P_{A}\left(R^{k-1} / R^{k}\right)=P_{A}\left(R^{k-1} / \operatorname{rad} R^{k-1}\right)=P_{A}\left(R^{k-1}\right)$. Similarly, $r_{B}^{k}=\operatorname{rad} r_{B}^{k-1}$, and then $Q=P_{B}\left(r_{B}^{k-1} / r_{B}^{k}\right)=P_{B}\left(r_{B}^{k-1}\right)$. Here we are using the condition $I \subseteq r_{B}^{2}$.

Since $A$ is hereditary, $R$ is projective as an $A$-module and we have $P_{A}\left(R^{k-1}\right)=$ $R^{k-1}$. Similarly $P\left(r_{B}^{k-1}\right)=r_{B}^{k-1}$, since $B$ is hereditary. Furthermore, $R^{k} \cong R \bar{P}$, $R^{k+1} \cong R^{2} \bar{P}$ as $A$-modules and $r_{B}^{k}=r_{B} Q$ and $r_{B}^{k+1}=r_{B}^{2} Q$ as $B$-modules. Therefore we have the following isomorphisms of $A$-modules:

$$
R^{k} / R^{k+1}=(R \bar{P}) /\left(R^{2} \bar{P}\right) \cong\left(r_{B} Q / I Q\right) /\left(r_{B}^{2} Q / I Q\right) \cong r_{B}^{k} / r_{B}^{k+1}
$$

If one tracks the isomorphism, one would find that the above isomorphism is actually induced from the quotient map $\pi: B \rightarrow A$ since $\bar{P}$ and $Q$ are subsets of $A$ and $B$ respectively (both algebras are hereditary).

Since $B$ is Artinian, we have $r_{B}^{m}=0$ for some $m$. If $I \neq 0$, there exists $p \geq 1$ minimal such that $I \nsubseteq r_{B}^{p}$. Then take $x \in I \backslash r_{B}^{p}$. It means that $x \in r_{B}^{p-1}$ and $\pi(x)=0 \in R^{p-1}$. However, $\pi: r_{B}^{p-1} / r_{B}^{p} \rightarrow R^{p-1} / R^{p}$ is an isomorphism. Hence, 
the image of $x$ in $r_{B}^{p-1} / r_{B}^{p}$ has to be zero, i.e., $x \in r_{B}^{p}$, which contradicts the choice of $x$. Thus we must have $I=0$. This proves the result.

Theorem 6.5. Let $A$ be a hereditary Artinian algebra splitting over its radical such that the surjective homomorphism $\pi: k\left(\Delta_{A}, \mathcal{A}\right) \rightarrow A$ in Theorem 3.4 possesses the kernel $\operatorname{ker}(\pi) \subseteq J^{2}$. Then, $\pi$ is an isomorphism.

Proof. By Proposition 6.3, $\Delta_{A}$ is finite and acyclic. Then, $k\left(\Delta_{A}, \mathcal{A}\right)$ is finite dimensional and $\operatorname{rad} k\left(\Delta_{A}, \mathcal{A}\right)=J$ is the ideal generated by all $\mathcal{A}$-paths of length 1 . Thus, the condition in Proposition 6.4 is satisfied for the natural homomorphism from $k\left(\Delta_{A}, \mathcal{A}\right)$ to $A$. It follows that $A \cong k\left(\Delta_{A}, \mathcal{A}\right)$.

In Theorem 6.5 $A / r_{A}$ is not usually a separable algebra. Hence, this result can be thought of as an improvement of [DK, Theorem 8.5.4].

\section{ACKNOWLEDGEMENT}

The authors take this opportunity to express their thanks to B.M. Deng, M.M. Zhang, Y.B. Zhang and H.Y. Zhu for their helpful conversations and suggestions.

\section{REFERENCES}

[ASS] I. Assem, D. Simson and A. Skowronski, Elements of the Representation Theory of Associative Algebras Vol I: Techniques of Representation Theory, London Mathematical Society Student Texts 65, Cambridge University Press, Cambridge, 2006. MR.2197389 (2006j:16020)

[ARS] M. Auslander, I. Reiten and S. O. Smalø, Representation Theory of Artin Algebra, Cambridge University Press, Cambridge, 1995. MR.1314422 (96c:16015)

[B] K. Bongartz, A geometric version of the Morita equivalence. J. Algebra 139 (1991), no. 1, 159-171. MR1106345 (92f:16008)

[CL] F. U. Coelho and S. X. Liu, Generalized path algebras, pp. 53-66 in Interactions between ring theory and repersentations of algebras (Murcia), Lecture Notes in Pure and Appl. Math, 210, Marcel-Dekker, New York, 2000. MR1758401 (2001c:16027)

[DR] V. Dlab and C.M. Ringel, Indecomposable representations of graphs and algebras, Mem. Amer. Math. Soc. 6 (1976), no. 173, MR0447344 (56:5657)

[DK] Y. A. Drozd and V. V. Kirichenko, Finite Dimensional Algebras, Springer-Verlag, Berlin, 1994. MR 1284468 (95i:16001)

[HGK] M. Hazewinkel, N. Gubareni and V. V. Kirichenko, Algebras, Rings and Modules, Vol. 1, Mathematics and Its Applications Vol. 575, Kluwer Academic Publishers, New York, 2005. MR2106764 (2006a:16001)

[KY] M. Kontsevich and Y. Soibelman, Notes on $A_{\infty}$-algebras, $A_{\infty}$-categories and noncommutative geometry, Homological mirror symmetry, 153-219, Lect. Notes in Phys., 757, Springer, Berlin, 2009. MR2596638 (2011f:53183)

[Lam] T. Y. Lam, A First Course in Noncommutative Rings, Graduate Texts in Mathematics 131, Springer-Verlag, New York, 1991. MR.1125071 (92f:16001)

[Li] F. Li, Characterization of left Artinian algebras through pseudo path algebras, J. Australia Math. Soc., 83 (2007), 385-416. MR2415878 (2009f:16022)

[LC] F. Li and L. L. Chen, The natural quiver of an Artinian algebra, to appear in Algebras and Representation Theory, online, 2010.

[LW] F. Li and D. W. Wen, Ext-quiver, AR-quiver and natural quiver of an algebra, in Geometry, Analysis and Topology of Discrete Groups, Advanced Lectures in Mathematics 6, Editors: Lizhen Ji, Kefeng Liu, Lo Yang, Shing-Tung Yau, Higher Education Press and International Press, Beijing, 2008.

[Liu] G. X. Liu, Classification of finite dimensional basic Hopf algebras and related topics, Doctoral Dissertation, Zhejiang University, China, 2005. 
[P] R. S. Pierce, Associative Algebras, Springer-Verlag, New York, 1982. MR674652 (84c:16001)

[R] C.M. Ringel, Representations of $K$-species and bimodules, J. Algebra 41 (1976), no. 2, 269-302. MR0422350(54:10340)

Department of Mathematics, Zhejiang University, Hangzhou, Zhejiang 310027, PeoPle's RePublic of China

E-mail address: fangli@cms.zju.edu.cn

Department of Mathematics, Kansas State University, Manhattan, Kansas 66506

E-mail address: zlin@math.ksu.edu 